\title{
The Diffusion of Crisis-Related Communication on Social Media: An Empirical Analysis of Facebook Reactions
}

\author{
Björn Ross*, Tobias Potthoff*, Tim A. Majchrzak ${ }^{\dagger}$, Narayan Ranjan Chakraborty ${ }^{\dagger}$, Mehdi Ben Lazreg ${ }^{\dagger}$, Stefan Stieglitz* \\ *University of Duisburg-Essen, Germany \\ Email: \{bjoern.ross; tobias.potthoff; stefan.stieglitz\}@uni-due.de \\ ${ }^{\dagger}$ University of Agder, Kristiansand, Norway \\ Email: \{timam; narayan.chakraborty; mehdi.ben.lazreg\}@uia.no
}

\begin{abstract}
During a crisis, authorities need to effectively disseminate information. We address the problem of deciding how crisis-related information should be published on Facebook to reach as many people as possible. We examine three recent terrorist attacks in Berlin, London and Stockholm. Our specific focus lies with official Facebook pages by municipalities and emergency service agencies. We collected posts about the events, along with the number of shares, likes and emotional reactions to them. In a regression analysis, several variables were examined that capture decisions on which information to publish and how. Posts containing condolences were found to result in three times as many emotional reactions as other posts, all other variables held constant. Images and videos positively affected the number of reactions by factors of 2.2 and 3.9, respectively, while text length negatively affected the number of shares. These results will help in the development of effective guidelines.
\end{abstract}

\section{Introduction}

When people are affected by crises such as terror attacks, they turn to social media to make sense of the situation [16]. Emergency service agencies (ESAs) have recognised an opportunity to rapidly disseminate information to many people and interact with them [31]. Consequently they have set up Twitter accounts and Facebook pages [54]. However, it is unclear which factors influence the success of their posts. To ensure effective emergency management practices, it is necessary to understand how individuals react to crisisrelated information. In particular, it is essential to learn what kind of information diffuses on social media.

In this article, we argue that while previous research has looked at the quantity of interactions, the relationships between qualitatively different interactions have received comparatively little attention. Examples on popular social media services include replies versus retweets on Twitter, or Facebook shares versus likes. Our assessment especially applies to the context of crisis management. In addition,
Facebook recently introduced a feature that allows users to express their views on a post by clicking an emoji [28]. This data could be highly relevant for emergency management: it would help responders understand how individuals react to their posts.

To evaluate the use of such user response data in emergency management, we studied how several variables related to a post affect the number of times it is shared and liked on Facebook. For this purpose, we collected posts by emergency management agencies and municipalities during three recent terror attacks, along with the reactions to them. The attacks examined were (1) the December 2016 Berlin truck attack, (2) the March 2017 attack in Westminster, London, and (3) the 2017 April Stockholm attack. Among the reactions collected were the number of shares and likes, and a range of other emotional reactions including sadness and anger.

Several variables were extracted that capture decisions made by page administrators and content creators on how to publish the information. This allowed us to examine their relative importance in determining user reactions. We also scrutinised the relationships among qualitatively different emotional reactions expressed on Facebook by using its new Reactions feature, and discuss its relevance for emergency management.

Our work makes several contributions. First, we provide comprehensive work on social media analytics in the field of crisis management with a particular focus on Facebook. By both exploring the topic theoretically and presenting an empirical study, we seek to advance the understanding of reactions to postings. Second, we discuss our findings with the aim of providing generalisable insights. Thereby, we provide advice for practitioners and contribute to the theory on social media analytics. Moreover, we hope to stimulate more work in this relatively unexplored area.

The remainder of this paper is structured as follows. In Section 2 we explain the background of the tackled topics. Due to the multi-step nature of our work, we describe our research design in detail in Section 3 The results of our study are then given in Section 4 . We discuss them in Section 5 before drawing a conclusion in Section 6 . 


\section{Background}

\subsection{Crisis Communication in Emergency Response}

In every emergency situation, and even more so in every crisis, communication is vital [21], [24]. To effectively mitigate the consequences of incidents, crisis managers (e.g. from a municipality or the police) must coordinate first responders and subsequent crisis stakeholders. Ineffective communication is at least one of several contributing factors to failed crisis response [38]. Good communication not only leads to a quicker and more effective crisis response, but also helps to avoid problems such as responders being deployed to a site which others have already been deployed to, possibly leaving another site uncovered.

Communication, however, does not only aid the response process. Equally, it can make the response easier, reduce suffering, and possibly even directly mitigate crisis consequences [47]. For this purpose, communication not only happens between crisis responders but also between crisis managers (or, in general, authorities) and the population [18]. If citizens know which kind of crisis they face and if they are provided with reliable information that helps them make decisions, they are much less likely to encounter trouble.

Crisis communication can happen on several levels. Before a crisis, the population can already be instructed. Imagine a region is prone to flooding. It helps noticeably if the population is taught about good procedures in case of a flood, such as avoiding cellars, switching off electricity, and looking after elderly neighbours. During crises, the population can be kept informed with updated news and with advice. Crises are chaotic, particularly in their onset [7]; therefore, a hybrid strategy of giving instructions (such as encouraging people to evacuate an area) and providing information to calm the population while supporting their own informed decisions is promising.

There are different ways of reaching the population with technological means. Traditionally, radio broadcasting was the main way of informing the public [36]. Currently, short messages (SMS) are popular for crisis communication due to the widespread usage of mobile phones [8]. It is not only possible to broadcast messages to all members of a particular population but also to all mobiles phones in an area, i.e. also including visitors [10]. Moreover, more targeted sending is possible, to e.g. give instructions to people registered as volunteer helpers. The Web and, recently, social media services complement the two older possibilities (cf. e.g. [48], [55]). They are arguably more vulnerable to infrastructure failures. Consider, for example, an earthquake. It is quite likely that parts of the electrical and the communication infrastructure are damaged; in this case, Internet access (or rather communication modes in general [49]) may be seriously disrupted. It takes much more damage before the cellular network is completely inoperable. Radio remains an option even in cases of the most serious damages. However, the higher level of media richness, personality, and in particular the social aspect are strong reasons for using several channels in crisis communication, as will become apparent throughout the remainder of this paper (cf. also [1], [18]).

\subsection{The Role of Social Media}

Since the advent of social media, its usage has rapidly increased. Social media platforms generally allow a two-way communication between individuals, groups, organisations and (emergency) authorities [58]. Because of social media's capabilities for mass communication, the rapid spread of information and the large potential audience, it has become an important channel for marketers and others [12]. Some studies have looked into the dissemination of information on social media and showed that emotionally charged text yields stronger reactions and a faster dissemination throughout a network [52]. For example, hate speech has become a problem on social media [44].

However, social media has also become an intermediary for news and other kinds of information due to the fact that people often consult the platforms' timelines, i.e. an aggregated view on postings based on the user's preferences, for updates of newspapers or other information providers [19], [29]. Valuable information is generated in social media - not only messages or pictures but also by users sharing location data, interests and relationships and giving likes or other reactions to original posts [11]. This initiates a backchannel for organisations which have started to listen to users' needs through social media or implemented customer service [26].

2.2.1. Social Media in Crises. In crisis situations, social media has gained importance as both an information source and a communication channel. Several studies have empirically examined how social media was used in different crisis scenarios: e.g. corporate brand crises [27], natural disasters [42], [5], riots and demonstrations [14] or acts of terrorism [45], [34], [9]. For example, Yin et al. [58] used natural language processing and data mining techniques in their study in order to enhance situational awareness. This concept from the military domain has also been adapted for non-military crisis situations. Based on Twitter data from eight crisis situations in Australia, including both humanmade and natural disasters, they developed an incident detection, clustering and visualisation system for emergency agencies. A more recent study investigated the Twitter communication during the 2013 Boston Marathon bombings, the 2014 hostage crisis in Sydney and the Charlie Hebdo attack in 2015 [4]. The results remain rather descriptive but indicate a general increase in the number of tweets and a 
higher proportion of retweets and hashtags during a crisis. Furthermore, they found accounts by the police and news media to have a strong influence on the communication.

Studies evaluating and consequently recommending communication strategies of crisis authorities in social media are scarce. Carter et al. [5] found that agencies were not really listening to crisis-related communication in social media nor did they respond to comments.

Because any user can publish posts on social media, there are also rumours and misinformation in the communication [37], [2]. Though Jong [25] found a self-correction mechanism in the context of a Dutch crisis, the identification of rumours and wrong information remains a topic of high interest which needs to be considered when using social media data.

2.2.2. Twitter Bias in Research. Most works aiming at the usage of social media data in the context of crisis communication and emergency response have only looked at Twitter. In general, there seems to be a bias towards Twitter in research on social media analytics and related fields. For example, in a study of humanitarian information processing, Munro and Manning [35] found that Twitter accounted for only $0.16 \%$ of messages sent globally, but for $74.29 \%$ of research papers published in the three databases examined over the course of one year. In this regard, the value of statistically valid predictions based on Twitter data has been challenged [17]. Although there are many reasons to use Twitter as an information source in crisis situations and in social media analytics in general (e.g. short messages, tweets are public by default, detailed API), we need to critically reflect on how well tweets mirror reality [46], [32]. As of January 2017, Facebook has 1,871 million active users whereas Twitter states 317 million only [6].

A comparative study by Eriksson and Olsson [15] investigated the perception of both Twitter and Facebook with professionals from ESAs and citizens in Sweden. They found that ESAs feel more experienced with Facebook than Twitter though its usage is rather low. Additionally, Twitter was considered an elite channel used to reach journalists or decision-makers, whereas Facebook can reach the general public. Citizens that act as recipients and co-creators onsite also valued Facebook higher than Twitter [15]. For example, Facebook was used during the European Floods of 2013 to organise volunteer communities [43]. Focusing on the communication sent by fire and police departments during the 2012 Hurricane Sandy, Hughes et al. [22] found relatively few departments using social media to inform and encourage a certain behaviour. They performed a broad usage analysis of different social media and coded the retrieved communication (e.g. closure, safety, weather). The number of reactions (e.g. likes) or shares to assess the potential reach were not included. Though the paper concludes with general recommendations on how to integrate social media in emergency response management, these remain rather high-level.

2.2.3. Facebook Reactions. In February 2016, after a pilot test in several markets, Facebook made the new Reactions feature available globally [28]. This feature allows users to react to posts by clicking an emoji displayed in the user interface alongside the previously available 'like' button. The reactions introduced are 'love', 'haha', 'wow', 'sad', and 'angry'. The new distinction has not yet been used in many studies. Larsson analysed the frequency of reactions, and the association between reactions and shares or comments, respectively [30]. The author concludes that news which provoke negative reactions get shared and commented on more, while news with many positive reactions (e.g. love) get shared and commented on less. In another study about the new reactions, the predictability of emotions based on texts of Facebook posts was studied [39]. They used the reactions to a Facebook post as labels in a supervised machine learning task, obviating the need for human annotations.

\subsection{Emergency Service Agencies}

Emergency Service Agencies (ESAs) are the prime responders to any crisis happening throughout the world. They range from police, NGOs, and fire brigades to other municipal authorities or joint agencies for larger areas or whole countries. Due to the critical aspect of the messages shared by ESAs which can help save lives and infrastructures, it is important that messages reach as many people as possible. Social media have changed the scope of ESAs' activities as these media allow to reach many people timely and efficiently. They can further enable a multi-way informationsharing platform for all the parties involved in the crisis. In contrast to the old channels which audiences need to switch on deliberately, social media are ubiquitous through mobile devices and push notifications [3]. As an example, during Hurricane Sandy, ESAs used social media to update the public about the situation, to issue evacuation orders and to answers people's concerns as many affected persons used social media as a lifeline [22]. Moreover, infrastructure providers such as water, power and transportation organisations used social media to share updates on the availability of their services during crises.

However, after several interviews with public sector emergency managers, Hiltz et al. [20] identified a lack of a comprehensive guideline as the main reason prohibiting ESAs from using social media more effectively. Nevertheless, research has developed useful though abstract recommendations for the usage of social media in ESAs (e.g. in [22], [5]). Hence, ESAs have started to use social media as source for enhancing situational awareness with on-site information. But the information flow in social media is updated constantly. Thus it is very difficult to get the overall

Page 2527 
picture while substantial portions of the data are redundant [40]. Another challenge for ESAs is to make decisions based on information with unknown credibility (e.g. rumours, fake news) from both official and unofficial sources [50], [37].

For the dissemination of important information, ESAs now actively use social media to reach a large proportion of the population faster [13]. Again, some effort has been made in the direction of producing a guideline. For example, The Contribution of Social Media In Crisis Management (COSMIC) is a EU-funded project to identify how social media can be most effectively used in crisis situations. They propose key steps ESAs should follow to publish information on social media before, during and after a crisis [23]. They put an emphasis on identifying the target group, writing specifically for that group, and being concise. Wendling et al. [57] pointed out that building trust and communicating with the public during normal times is important because people will always look for familiar sources of information during a crisis.

Besides the mentioned two-way communication, social media allows its users to get feedback about the perceptions of posts and tweets. Reactions such as comments, retweets, likes or the aforementioned 'haha', 'sad' etc. on Facebook can serve as a proxy for the success of the ESAs' posts. Since reactions increase the reach of posts, there might be an interest in formulating posts in a way that more reactions and, consequently, a higher reach are achieved. Quantitative studies investigating the effects of writing style or embedded media on reactions could not be identified. We aim at filling that gap and analyse Facebook posts from during three different crises.

\section{Research Design}

\subsection{Data Collection}

We revisited previous studies in the realm of crisis communication and emergency response that use social media data. Above we argued that there is a bias towards the use of Twitter in studies on social media analytics in general, and on crisis communication and response in particular. Hence, we focus on the social networking site Facebook.

Additionally, prior work analysed communication during crises to identify roles and networks, cluster topics or describe sense-making processes through categories of information. In this study, we focus on ESAs' communication strategies with regard to their achieved reactions. Furthermore, we innovatively take the different reactions available on Facebook into account.

Three recent crises were selected for the study: (1) the 2016 Berlin attack when a truck was deliberately driven into a Christmas market at the Breitscheidplatz on 19 November 2016, (2) the $\mathbf{2 0 1 7}$ Westminster attack when

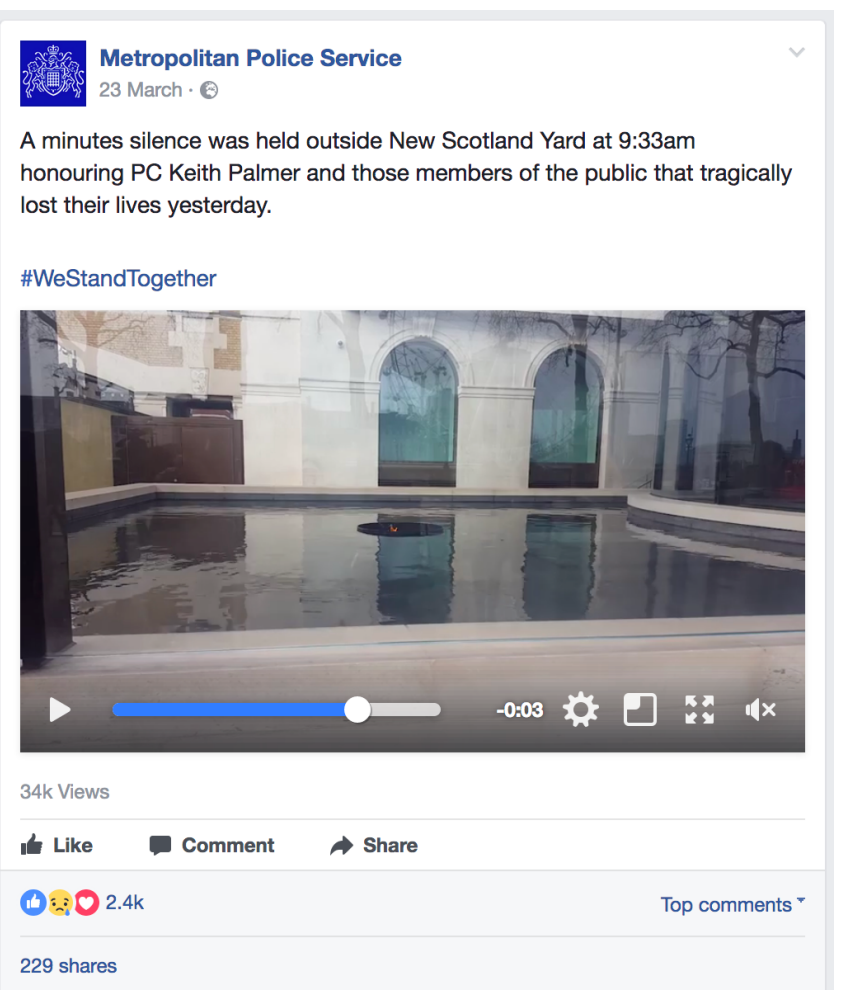

Figure 1: Example Facebook post made by the London Metropolitan Police Service shortly after the Westminster incident

a car was deliberately driven into pedestrians on the Westminster bridge and a police officer stabbed, on 22 March 2017, and (3) the 2017 Stockholm attack when a truck was deliberately driven into crowds at the shopping street Drottninggatan, on 7 April 2017. The crises were selected due their similarity, since they were all classified as acts of terrorism. They took place in three different countries, all in Europe.

We adopted the Social Media Analytics Framework of Stieglitz et al. [53] which has also guided other research. The model foresees the phases (1) Tracking (using APIs or other parsing techniques), (2) Preparation (e.g. removing spam, coding) and (3) Analysis.

A total of 85 Facebook posts were collected from six relevant Facebook pages (see Table 1 for an overview, and Figure 1 for an example). We started the data collection by manually searching for public Facebook pages of authorities involved in the official communication. The search was carried out using the Facebook search box, e.g. by searching for 'Police London', and by checking whether the official websites of municipalities and ESAs provided hyperlinks to corresponding Facebook pages. For each incident, we selected one Facebook page run by an ESA and one run by a municipality. In the case of London and Berlin, the ESA involved is the official police service; in the case 
Table 1: Facebook pages examined

\begin{tabular}{lrrr}
\hline & Berlin & London & Stockholm \\
\hline $\begin{array}{l}\text { Munici- } \\
\text { pality }\end{array}$ & $\begin{array}{r}\text { Berlin.de } \\
\text { (@ Hauptstadt- } \\
\text { portal) }\end{array}$ & $\begin{array}{r}\text { London Gov } \\
\text { (@LDNGov) }\end{array}$ & $\begin{array}{r}\text { Stockholms stad } \\
\text { (@ sthlmsstad) }\end{array}$ \\
$\begin{array}{lrrr}\text { Likes* } \\
\text { Posts }\end{array}$ & 41063 & 55230 & 876 \\
\hline ESA & 2 & 3 & 15 \\
& Polizei Berlin & MPS & $\begin{array}{r}\text { Kris- } \\
\text { information.se } \\
\text { (@ Kris- } \\
\text { information) } \\
\text { Likes* }\end{array}$ \\
Posts & (@Polizei- & (@ metpoliceuk) & 45281 \\
\hline Time span & Berlin) & 165429 & 37 \\
\hline
\end{tabular}

*Page likes as of 12 Jun 2017

of Stockholm, the Swedish Civil Contingencies Agency, an official government agency, runs the website Krisinformation.se along with a corresponding Twitter account and Facebook page. This page publishes information about ongoing and imminent crises as well as information about crisis management.

We collected all posts published on these pages within seven days of the incident in May 2017. Related numbers (e.g. likes and other reactions as well as shares and video views if applicable) were gathered accordingly. Large numbers were rounded due to a limitation in the Facebook user interface. Due to the time ( 1 to 4 months) between the crises and the data collection, we expect the posts' reactions to have stabilised. Whether a post contained pictures or videos was also noted.

\subsection{Data Preparation}

To allow a detailed analysis of the kind of information and the style used in the ESAs' posts, we annotated all collected posts. Prior work has proposed categories in the context of a natural crisis [22] or riots [14]. The former is, however, too specific for a weather-related crisis (e.g. cleanup, closures, damage, donations etc.). The second paper [14] was used as a basis. It also provides detailed genres such as demonstration start or number of participants below a top-level genre information.

We checked whether the proposed top-level genres information, encourage behaviour and warning can sufficiently categorise the posts. The initial screening of the posts suggested to add the genres number of victims and condolences besides the general genre information.

Lastly, the annotation process included the question whether a post was related to the crisis. Three researchers independently evaluated and annotated the texts.

After the annotation, we calculated the reliability of the annotations using Krippendorff's alpha (see Figure 2). The variable information was excluded because of its low reliability $(\alpha=0.237)$. The other variables $(\alpha>0.6)$ were

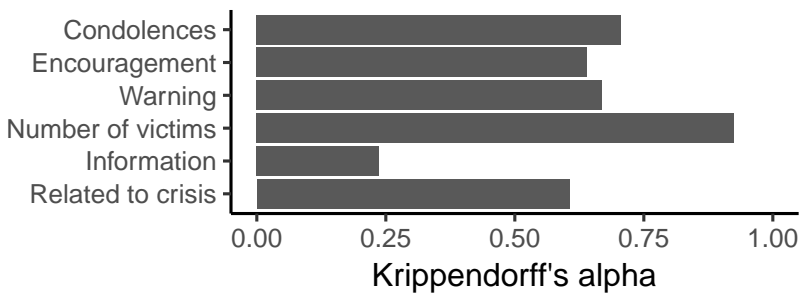

Figure 2: Reliability of the annotation (84 elements, 3 annotators each)

retained and converted to binary using a majority vote: a post is taken to contain condolences, for example, if at least two out of three annotators considered it to.

The data set was cleaned for the data analysis. One post was excluded from the analysis because it did not contain any text that could be annotated. Twelve posts were excluded because they were not considered relevant by at least two out of three raters. The pruned data set contained only three posts from the Greater London government page, and two from the Berlin Facebook page. These were removed due to the low case numbers. Finally, three posts were excluded for being shares of posts published by a different Facebook page. These posts had to be excluded from the analysis because, when a Facebook page shares a post from a different page, future shares are counted towards the original post. As a result, it is not possible to distinguish between the number of times a post was shared due to the ESA's followers actually seeing the post and the number of times it was shared by other Facebook users, including the original page's followers. The final data set contained 66 posts which were used in the analysis. Almost all of the posts were in the primary language of the respective country, the only exception being seven posts from Sweden in English, which is very widely understood there. Table $2 \mathrm{a}$ shows descriptive statistics for the final data set.

\subsection{Data Analysis}

Negative binomial regressions were calculated to explain the number of shares and the number of reactions to the Facebook posts based on the Facebook page the post appeared on, text length, the presence of an image, the presence of a video, and the reliable categories described above. All calculations were carried out using the statistical software package $\mathrm{R}$ [41] and the R package MASS [56].

Compared with ordinary least squares regression, generalised linear models allow for error distributions other than the normal distribution. In this case, the residuals are assumed to be from a negative binomial distribution, which is appropriate for a non-negative integer response in the presence of overdispersion.

The log link function was used, i.e,

$$
\log \left(\hat{y}_{i}\right)=x_{i}^{T} \beta \Longleftrightarrow \hat{y}=\exp \left(x_{i}^{T} \beta\right),
$$


Table 2: Descriptive statistics of the variables for the cleaned sample $(n=66)$

(a) Numerical variables

\begin{tabular}{lrrrrrrr}
\hline Variable & Min & $1 \mathrm{Q}$ & Median & 3Q & Max & Mean & $\%$ Zero \\
\hline Likes & 3 & 57.5 & 174.5 & 693.2 & 13000 & 737.70 & 0.0 \\
Sadness & 0 & 2 & 19 & 112 & 7400 & 287.50 & 21.2 \\
Angry & 0 & 0 & 1 & 6 & 525 & 21.98 & 45.5 \\
Haha & 0 & 0 & 0 & 0 & 59 & 1.15 & 83.3 \\
Love & 0 & 0 & 5 & 22.3 & 733 & 51.12 & 31.8 \\
Wow & 0 & 0 & 0 & 1 & 28 & 2.08 & 62.1 \\
\hline All reactions & 3 & 57.5 & 174.5 & 1145 & 21231 & 1101.60 & 0.0 \\
\hline Shares & 0 & 24.5 & 89.5 & 299 & 7501 & 495.35 & 4.5 \\
Text length (in characters) & 42 & 134 & 234 & 822.8 & 5816 & 649.80 & 0.0 \\
\hline
\end{tabular}

(b) Binary variables

\begin{tabular}{lr}
\hline Variable & \% Zero \\
\hline Image & 69.7 \\
Video & 92.4 \\
Number of victims & 92.4 \\
Warning & 93.9 \\
Encouragement & 57.6 \\
Condolences & 75.8 \\
\hline
\end{tabular}

where $\beta$ is the parameter vector, $x_{i}$ is the vector of predictors for observation $i$, and $\hat{y}_{i}$ is the conditional mean of the response given $x_{i}$. The predictors are thus expected to be related linearly to the logarithm of the response, instead of directly to the response. In other words, the addition of an image to a post is not expected to increase its number of likes by an absolute number, whether the post has 10 or 10,000 likes. Instead, the image is expected to increase the number of likes by a percentage. Likewise, the variable text length was log-transformed with base 2 because a percent change in the text length is assumed to be associated with a percent change in the number of shares and reactions.

Dummy variables were introduced to control for the effect of the page on which the post was published. The number of reactions to a post is likely to depend to a large degree on circumstances outside the immediate control of the page owner at the time the information is posted. These may include differences in media consumption habits between different countries and between police and municipality pages, as well as in the number of followers. Conceptually, the dummy variables are statistical control variables. Their inclusion allows us to neutralise the effects of all such variables that differ between pages, but do not differ between posts on the same page.

In addition to the regression analyses carried out to explain the differences in the sum of all reactions as well as shares, we also examined the correlation coefficients between the individual reaction types (e.g. sadness, anger). Finally, we exemplarily studied the posts with the highest proportion of each reaction to understand which types of content result in which emotional reactions.

\section{Results}

\subsection{Regression Analysis}

To study how post content and other variables affect the diffusion of Facebook posts, we calculated a negative binomial regression as described above. The results are shown in Table 3 The exponentiated coefficients give a ratio for the change in the expected number of reactions or shares when the independent variable is increased by 1 , all other variables held constant. In the case of the binary variables, this corresponds to a change from absence to presence (of an image, encouragement, etc.). In the case of text length, it corresponds to a doubling of text length.

The Facebook page that a post was published on has a strong influence on the results. For example, a post on the page of Polizei Berlin is expected to accrue 251 times as many shares, and 158 times as many reactions, as a post on the City of Stockholm page (the reference category).

However, the results also show that other variables influence the results. The text length is a major contributor to the number of shares, since doubling the text length leads to a decrease in the number of shares by a factor of 0.68 , or $32 \%(p=.011)$.

In contrast, the number of reactions is greatly influenced by whether or not the post contains media. If it contains an image, the number of reactions increases by a factor of $2.16(p=0.038)$. The effect of a video is even stronger, as it increases the number of reactions by a factor of 3.89 $(p=0.020)$.

Finally, the number of emotional reactions is also governed to a large degree by whether or not the post expresses condolences or reports the expression of condolences by a third party. If it does, the number of reactions is increased by a factor of $2.99(p=0.001)$.

As for the other variables, these results are inconclusive. It is not clear whether the other categories such as warning messages and encouragement have any effect at all on either the number of shares or reactions, whether text length influences emotional reactions, or whether images and video influence shares. All of these variables could have a large influence, as evidenced by the size of the estimated coefficients, but the standard errors and corresponding $p$ values are large, so any judgment is best suspended based on the available data. 
Table 3: Results of the negative binomial regression models $(\mathrm{n}=66)$

\begin{tabular}{|c|c|c|c|c|c|c|c|c|c|c|}
\hline \multirow[b]{2}{*}{ Variable } & \multicolumn{5}{|c|}{ Number of shares } & \multicolumn{5}{|c|}{ Number of reactions } \\
\hline & $\beta$ & $\exp (\beta)$ & SE & $\mathrm{Z}$ & $\mathrm{p}$ & $\beta$ & $\exp (\beta)$ & SE & $\mathrm{Z}$ & $\mathrm{p}$ \\
\hline (Intercept) & 5.20 & & 1.30 & 4.02 & $<.000^{*}$ & 3.72 & & 0.86 & 4.32 & $<.000^{*}$ \\
\hline \multicolumn{11}{|l|}{ Controls } \\
\hline Page Krisinformation.se & 2.57 & 13.10 & 0.58 & 4.43 & $<.000^{*}$ & 2.91 & 18.35 & 0.39 & 7.51 & $<.000^{*}$ \\
\hline Page Metropolitan Police London & 3.44 & 31.09 & 0.65 & 5.32 & $<.000^{*}$ & 3.50 & 33.21 & 0.43 & 8.18 & $<.000^{*}$ \\
\hline Page Polizei Berlin & 5.53 & 251.03 & 0.73 & 7.59 & $<.000^{*}$ & 5.06 & 158.36 & 0.48 & 10.47 & $<.000^{*}$ \\
\hline \multicolumn{11}{|l|}{ Explanatory variables } \\
\hline $\log ($ Text length $)$ & -0.39 & 0.68 & 0.15 & -2.55 & $.011^{*}$ & -0.14 & 0.87 & 0.10 & -1.42 & .157 \\
\hline Image & 0.47 & 1.59 & 0.56 & 0.83 & .405 & 0.77 & 2.16 & 0.37 & 2.07 & $.038^{*}$ \\
\hline Video & 1.22 & 3.40 & 0.88 & 1.40 & .163 & 1.36 & 3.89 & 0.58 & 2.33 & $.020^{*}$ \\
\hline Number of victims & 0.59 & 1.81 & 0.76 & 0.78 & .434 & 0.59 & 1.81 & 0.50 & 1.18 & .238 \\
\hline Warning & 1.00 & 2.71 & 0.76 & 1.32 & .188 & 0.43 & 1.53 & 0.50 & 0.85 & .398 \\
\hline Encouragement & 0.37 & 1.44 & 0.44 & 0.84 & .404 & -0.21 & 0.81 & 0.29 & -0.71 & .478 \\
\hline Condolences & 0.50 & 1.65 & 0.52 & 0.97 & .333 & 1.10 & 2.99 & 0.34 & 3.19 & $.001^{*}$ \\
\hline
\end{tabular}

${ }^{*} p<.05$

Table 4: Correlation matrix for Facebook reactions

\begin{tabular}{lrrrrrr}
\hline & Likes & Sadness & Angry & Wow & Haha & Love \\
\hline Shares & .785 & .731 & .334 & .469 & .275 & .526 \\
Likes & & .935 & .149 & .426 & .175 & .859 \\
Sadness & & & .342 & .473 & .041 & .748 \\
Angry & & & & .639 & .224 & .009 \\
Wow & & & & & .526 & .210 \\
Haha & & & & & & .002 \\
\hline
\end{tabular}

\subsection{Relationships between Facebook Reactions}

The new Facebook Reactions feature offers the possibility to provide unique insights regarding the quality of emotions experienced by Facebook users as they consume content. To study the relationships between these different emotional reactions, we calculated the correlation coefficients between the Facebook reactions (see Table 4 ).

Upon inspecting the results, it becomes clear that most of the reactions are related. All correlation coefficients are positive. However, some pairs exhibit very high correlation coefficients, such as like and love $(r=.859)$, but also like and sadness $(r=.935)$. Other pairs appear to be unrelated, such as sadness and haha $(r=0.041)$, or angry and love $(r=.009)$, meaning that they are used in response to different posts.

To better understand the types of content that resulted in certain types of reactions, we examined the posts with the highest proportion of each reaction. These posts may exemplify the kind of content typical for a specific emotion.

The very first post by Polizei Berlin after the attack was a factual statement in which they informed the public about the attack: 'On the evening of 19.12.2016 a truck was driven over the walkway at \#Breitscheidplatz in people on the Christmas market ...' (19 Dec 2016, 9:29 PM). Later the post was updated to include the fact that 12 people had been killed and to encourage the public to share information with them via telephone. This post was the one with the highest proportion of reactions of sadness indicated $(61.6 \%$ of reactions expressed in response to this post). This number is noteworthy because in almost all other cases the most frequent reaction was a 'like', but not here.

In the post that made the most users angry, as measured by their use of the Reactions feature $(29.7 \%$ were clicks of the 'angry' emoji), the Metropolitan Police informed the public about the identity of the attacker and encouraged people to call an anti-terrorist hotline if they had information concerning him: 'Westminster Attack: Man believed responsible named ...' (23 Mar 2017, 4:49 PM).

Both of these very negatively received posts provided information that clearly linked the reported data to a terrorist incident. In contrast, in the post with the highest proportion of 'wow' reactions (2.1\%), Krisinformation.se bluntly asked its followers to avoid certain areas of the city: 'Police urge the public to avoid Sergels torg and the area around it in central Stockholm.' (7 Apr 2017, 3:52 PM).

The descriptive statistics in Table 2a made clear that negative reactions were, unsurprisingly, much more frequently experienced in response to crisis-related information than positive reactions. Nevertheless, some Facebook users saw positive aspects in the examined posts. In the post with the highest proportion of 'love' reactions $(16.6 \%)$, the Metropolitan Police reported a statement made by the family of a police constable who had died during the attack. They thanked the public and police for their support, stating that '... we have been overwhelmed by the love and support for our family, and most especially, the outpouring of love and respect for our Keith ...' (26 Mar 2017, 1:37 PM). A photo of the victim was attached to the post.

Finally, the German Police provided some much needed comic relief when they dispelled a rumour that a second attack at a shopping mall was imminent, saying '... Today the car park of the Gropius Passagen was closed twice. Once because of overfilling and once because of a broken-down car in the driveway ...' (23 Dec 2016, 5:55 PM). This post had the highest proportion of 'haha' reactions $(2.3 \%)$. 


\section{Discussion}

Our results show that post content influences both quantity and quality of user reactions on Facebook. Reactions were the most negative when the ESAs shared initial information at the onset of the crisis. The most positive reactions, however, were observed after the crisis was over, when the family of a deceased victim thanked the public for their support. These observations are indicative of sense-making patterns observed elsewhere in crisis communication [34].

Posts offering condolences could be shown statistically to result in more emotional reactions from Facebook users than non-emotional posts. This aligns with previous findings made in the context of other social media [52], [30].

These results have important practical implications for those operating the Facebook pages of municipalities and ESAs during crises. In this context, the number of likes and shares for a post are key indicators of a post's performance. The goal of the agencies concerned is to support community resilience by disseminating information, warnings and the encouragement of certain behaviour rapidly to as many people as possible. In contrast, the posts that really gain the most visibility in terms of likes and other reactions are those with emotional content, which are perhaps less immediately useful for accomplishing the operator's crisis response strategy. This is an apparent paradox: the goal of page owners may be to spread information, but Facebook users seem to be the most interested in emotional content. Carter et al. [5] also raised the question whether people prefer emotional support from peers or official information from agencies. Our results indicate that ESAs should take advantage of an emotional style while informing the public.

Our findings have other important implications for the Facebook pages of municipalities and ESAs. At a glance, the statistical results can be restated in the following way:

- Keep your posts concise. Doubling their length will decrease their number of shares by a third, all else equal.

- Use image and/or video along with text in your post. An image will approximately double user reactions, while a video will quadruple them.

- The most important factor, however, that determines both the number of shares and other reactions is the number of followers and other variables outside your immediate control. Prepare accordingly and encourage as many people as possible to subscribe to your updates by liking or following your page.

This kind of advice is of course not entirely new [23]. Suggestions to keep posts short and illustrate them are also being circulated on popular media such as blog posts by social media marketing agencies 1 . However, most of

1. For example, see https://blog.hubspot.com/marketing/how-to-writeblog-post-simple-formula-ht that evidence is anecdotal. Often it results from qualitative observations that have not sought to quantify the effect in an international, cross-cultural setting spanning several crises. Only rarely are these blog posts backed up by a published reproducible methodology.

The individual ESAs showed different communication behaviour. The Polizei Berlin and Krisinformation.se used shorter texts and appeared to follow some guidelines, whereas the Metropolitan Police London mostly posted long texts partially with direct quotes that seemed to be taken from their website. They may consider shortening their future posts if they want their posts to be shared more often. On the other hand, many posts of the Metropolitan Police London were coded as condolence which were shown statistically to affect the number of reactions. Though topics such as condolences, thanks of victims etc. are not the primary task of ESAs, their inclusion could motivate users to like the ESA page, which in turn will increase the organic reach in the next crisis situation. Thus, ESAs might want to consider developing ways to 'nudge' users to actively spread their information [33].

Our results also have intriguing implications for our understanding of information diffusion. There were substantial differences between the factors leading to more likes and more shares. Future research could examine the differences in decision processes between these forms of user interactions, and the sociological and psychological theories behind them. These results also mean that it is valuable to study several different forms of reactions in the same setting, since the findings may differ considerably.

Of course this research is not without limitations. Unlike the page owners, we do not have access to the actual reach of the posts or the number of impressions, but only to the number of shares and likes, which must serve as proxies and should be, for obvious theoretical reasons, very highly correlated with the variables of interest.

In our analysis of the reactions, we assumed that $\mathrm{Fa}$ cebook's Reactions feature makes the emotional response to a post measurable to some degree. However, the means reported in Table $2 \mathrm{a}$ show that only a fraction of Facebook users make use of this feature. Even if its use was more widespread, the five emojis offered could still only provide a rough indicator of actual emotional reactions. Ideally, the emotions experienced by users should be measured using a validated questionnaire or physiological response data although such an approach would, of course, be infeasible for such a large number of posts and users.

In this research, we also ignored the differences between Facebook pages by including them as dummy variables, which future research could examine these more closely. They replaced several omitted variables, such as the owner of the page, the number of followers, and differences in usage habits between countries, each of which could affect the outcome. Previous research has established that com- 
munication patterns differ considerably between crises [51]. Unsurprisingly, the dummy variables had large coefficients, which shows that they indeed had a strong influence on the results. Since these variables are outside the control of the page owner, they are unlikely to be of immediate interest to ESAs. However, they could still be of interest to the research community, and in the future could be examined individually. The language of a post may likewise affect its diffusion, especially when the post is in a minority language, and it will affect text length, especially when the languages are not closely related. Finally, the time of day and time of year an event occurs may affect reactions. To study these effects, posts will have to be collected from many more pages in a larger study that spans a longer period of time.

\section{Conclusion}

In this paper we argued that social media provide an opportunity for ESAs and other stakeholders to disseminate information more effectively by listening out to their reactions and optimising posts accordingly. It has become clear that page owners should not only care about how many users interact with a post, but also how they interact with it. The emotions they experience when perceiving the content play a role in determining how many people will see it and can benefit from it during the crisis. The possibilities offered by social media in this regard are becoming more and more detailed. If more users adopt Facebook's Reactions mechanism, page administrators will have a detailed picture of how audiences react to their content and an opportunity to fine-tune their information dissemination strategies accordingly. We showed several ways in which measurable user reactions are influenced by the content of a post, opening the door for further research in this area. The academic community can help by carrying out evidence-based research using published, reproducible methods that yield quantifiable results. The challenge is for stakeholders to closely monitor how their audiences react. If they succeed, this will increase their reach and contribute to the development of more effective emergency management practices.

\section{References}

[1] A. Acar and Y. Muraki, "Twitter for crisis communication: lessons learned from Japan's tsunami disaster," Int. J. Web Based Communities, vol. 7, no. 3, pp. 392-402, 2011.

[2] D. E. Alexander, "Social Media in Disaster Risk Reduction and Crisis Management," Science and Engineering Ethics, vol. 20, no. 3, pp. 717-733, sep 2014.

[3] S. Asur and B. Huberman, "Predicting the future with social media," in Proc. - 2010 IEEE/WIC/ACM Int. Conf. on Web Intelligence, WI 2010, vol. 1, 2010, pp. 492-499.

[4] C. Buntain, J. Golbeck, B. Liu, and G. LaFree, "Evaluating Public Response to the Boston Marathon Bombing and Other Acts of Terrorism through Twitter," in Proc. 10th ICWSM, 2016, pp. 555-558.
[5] L. Carter, J. B. Thatcher, and R. Wright, "Social Media and Emergency Management: Exploring State and Local Tweets," in Proc. 47th HICSS. IEEE, jan 2014, pp. 1968-1977.

[6] D. Chaffey, "Global social media research summary 2017," 2017. [Online]. Available: http://www.smartinsights.com/social-media-marketing/ social-media-strategy/new-global-social-media-research/

[7] J. Chan and T. Comes, "Innovative research design-a journey into the information typhoon," Procedia Engineering, vol. 78, pp. 52-58, 2014.

[8] T. W. Coombs, "Crisis management and communications," 2007. [Online]. Available: http://www.instituteforpr.org/ crisis-management-and-communications/

[9] J. Crump, "What are the police doing on twitter? social media, the police and the public," Policy \& Internet, vol. 3, no. 4, pp. 1-27, 2011.

[10] D. Barnes et al., "Public Warning," 2012. [Online]. Available: http://www.eena.org/uploads/gallery/files/ operations_documents/2012_06_25_publicwarning.pdf

[11] B. De Longueville, R. S. Smith, and G. Luraschi, “"omg, from here, i can see the flames!": A use case of mining location based social networks to acquire spatio-temporal data on forest fires," in Proc. Int.l Workshop Location Based Social Networks, ser. LBSN '09. ACM, 2009, pp. 73-80.

[12] L. De Vries, S. Gensler, and P. Leeflang, "Popularity of brand posts on brand fan pages: An investigation of the effects of social media marketing," J. Interactive Marketing, vol. 26, no. 2, pp. 83-91, 2012.

[13] L. A. S. Denis, A. L. Hughes, and L. Palen, "Trial by fire: The deployment of trusted digital volunteers in the 2011 shadow lake fire," in Proc. 9th ISCRAM, 2012.

[14] C. Ehnis, M. Mirbabaie, D. Bunker, and S. Stieglitz, "The role of social media network participants in extreme events," in Proc. 25th Austr. Conf. Inform. Syst., 2014.

[15] M. Eriksson and E.-K. Olsson, "Facebook and Twitter in Crisis Communication: A Comparative Study of Crisis Communication Professionals and Citizens," J. Contingencies and Crisis Management, vol. 24, no. 4, pp. 198-208, dec 2016.

[16] J. D. Fraustino, B. Liu, and J. Yan, "Social Media Use during Disasters: A Review of the Knowledge Base and Gaps," SMART, College Park, MD, Tech. Rep., 2012. [Online]. Available: https: //www.start.umd.edu/sites/default/files/files/publications/ START_SocialMediaUseduringDisasters_LitReview.pdf

[17] D. Gayo-Avello, "Don't turn social media into another 'Literary Digest' poll," Commun. ACM, vol. 54, no. 10, p. 121, oct 2011 .

[18] C. Grimes, M. Sakurai, V. Latinos, and T. A. Majchrzak, "Cocreating Communication Approaches for Resilient Cities in Europe: the Case of the EU Project SMR," in Proc. 14th ISCRAM. ISCRAM Assoc., 2017.

[19] A. Hermida, F. Fletcher, D. Korell, and D. Logan, "Share, like, recommend: Decoding the social media news consumer," Journalism Studies, vol. 13, no. 5-6, pp. 815-824, 2012.

[20] S. R. Hiltz and J. Kushma, "Use of Social Media by U.S. Public Sector Emergency Managers: Barriers and Wish Lists," in Proc. 11th ISCRAM, no. May, 2014, pp. 602-611.

[21] J. F. Home and J. E. Orr, "Assessing behaviors that create resilient organizations," Employment Relations Today, vol. 24, no. 4, pp. 29-39, 1997.

[22] A. L. Hughes, L. A. A. St. Denis, L. Palen, and K. M. Anderson, "Online public communications by police \& fire services during the 2012 Hurricane Sandy," in Proc. 33rd Ann. ACM CHI. ACM Press, 2014, pp. 1505-1514. 
[23] I. Helsloot et al., "Tips and tricks for citizens \& public authorities - How to use new media during crisis situations?" 2015. [Online]. Available: http://crisislab.nl/wordpress/wpcontent/uploads/Final-Guidelines_-Crisislab-website-1.pdf

[24] C. Johnson and S. Blackburn, "Advocacy for urban resilience: Unisdr's making cities resilient campaign," Environment and Urbanization, vol. 26, no. 1, pp. 29-52, 2014.

[25] W. Jong and M. L. Dückers, "Self-correcting mechanisms and echo-effects in social media: An analysis of the "gunman in the newsroom" crisis," Comp. in Human Behavior, vol. 59, pp. 334-341, jun 2016.

[26] A. M. Kaplan and M. Haenlein, "Users of the world, unite! The challenges and opportunities of Social Media," Bus. Horizons, vol. 53, no. 1, pp. 59-68, jan 2010.

[27] J. H. Kietzmann, K. Hermkens, I. P. McCarthy, and B. S. Silvestre, "Social media? Get serious! Understanding the functional building blocks of social media," Bus. Horizons, vol. 54, no. 3, pp. 241-251, may 2011.

[28] S. Krug, "Reactions Now Available Globally," 2016. [Online]. Available: https://newsroom.fb.com/news/2016/02/reactionsnow-available-globally/

[29] H. Kwak, C. Lee, H. Park, and S. Moon, "What is twitter, a social network or a news media?" in Proc. 19th Int. Conf. World Wide Web, ser. WWW'10. ACM, 2010, pp. 591-600.

[30] A. O. Larsson, "Diversifying Likes," J.ism Practice, no. January, feb 2017.

[31] M. Latonero and I. Shklovski, "Emergency Management, Twitter, and Social Media Evangelism," IJISCRAM, vol. 3, no. 4, pp. 1-16, 2011

[32] K. Leetaru, "Does Social Media Actually Reflect Reality?" Forbes, 2016. [Online]. Available: https://www.forbes.com/sites/kalevleetaru/2016/02/16/ does-social-media-actually-reflect-reality

[33] C. Meske and T. Potthoff, "The DINU model - A process model for the design of nudges," in Proc. 25th ECIS, 2017.

[34] M. Mirbabaie and E. Zapatka, "Sensemaking in Social Media Crisis Communication - A Case Study on the Brussels Bombings in 2016," in Proc. 25th ECIS, 2017.

[35] R. Munro and C. D. Manning, "Short message communications: users, topics, and in-language processing," in Proc. 2nd ACM Symp. Computing for Development, 2012.

[36] National Research Council, Public response to alerts and warnings on mobile devices: Summary of a workshop on current knowledge and research gaps. The National Academies Press, 2011.

[37] O. Oh, M. Agrawal, and H. R. Rao, "Community Intelligence and Social Media Services: A Rumor Theoretic Analysis of Tweets During Social Crises," MIS Quart., vol. 37, no. 2, pp. 407-426, 2013.

[38] J. O'Sullivan, R. Bradford, M. Bonaiuto, S. De Dominicis, P. Rotko, J. Aaltonen, K. Waylen, and S. Langan, "Enhancing flood resilience through improved risk communications," Nat. Hazard Earth Sys., vol. 12, no. 7, p. 2271, 2012.

[39] C. Pool and M. Nissim, "Distant supervision for emotion detection using Facebook reactions," in Proc. Workshop Computational Modeling of People's Opinions, Personality, and Emotions in Social Media, 2016, pp. 30-39.

[40] Y. Qu, C. Huang, P. Zhang, and J. Zhang, "Harnessing social media in response to major disasters," in CSCW 2011 Workshop, 2011.

[41] R Core Team, R: A Language and Environment for Statistical Computing, R Foundation for Statistical Computing, Vienna, Austria, 2016.
[42] J. Radianti, S. R. Hiltz, and L. Labaka, "An overview of public concerns during the recovery period after a major earthquake: Nepal twitter analysis," in Proc. 49th HICSS, Jan 2016, pp. 136-145.

[43] C. Reuter, T. Ludwig, M.-A. Kaufhold, and V. Pipek, "XHELP: Design of a Cross-Platform Social-Media Application to Support Volunteer Moderators in Disasters," in Proc. 33rd Ann. ACM CHI. ACM Press, 2015, pp. 4093-4102.

[44] B. Ross, M. Rist, G. Carbonell, B. Cabrera, N. Kurowsky, and M. Wojatzki, "Measuring the Reliability of Hate Speech Annotations: The Case of the European Refugee Crisis," in Proc. of NLP4CMC III, ser. Bochumer Linguistische Arbeitsberichte, vol. 17, Bochum, sep 2016, pp. 6-9.

[45] A. Ruggiero and M. Vos, "Terrorism Communication: Characteristics and Emerging Perspectives in the Scientific Literature 2002-2011," J. Contingencies and Crisis Manage., vol. 21, no. 3, pp. 153-166, sep 2013.

[46] D. Ruths and J. Pfeffer, "Social media for large studies of behavior," Science, vol. 346, no. 6213, pp. 1063-1064, 2014.

[47] M. Sakurai and R. T. Watson, "Securing communication channels in severe disaster situations - Lessons from a Japanese earthquake," in Proc. 12th ISCRAM, L. Palen, M. Buscher, T. Comes, and A. Hughes, Eds. University of Agder (UiA), 2015, pp. 288-299.

[48] N. Serrano, J. Hernantes, T. A. Majchrzak, and M. Sakurai, "Resilience information portal," in Proc. 14th ISCRAM. ISCRAM Assoc., 2017.

[49] S. C. Simmons, T. A. Murphy, A. Blanarovich, F. T. Workman, D. A. Rosenthal, and M. Carbone, "Telehealth technologies and applications for terrorism response: a report of the 2002 coastal north carolina domestic preparedness training exercise," J. Amer. Medical Informatics Assoc., vol. 10, no. 2, pp. 166-176, 2003.

[50] T. Simon, A. Goldberg, D. Leykin, and B. Adini, "Kidnapping whatsapp-rumors during the search and rescue operation of three kidnapped youth," Comput. in Human Behavior, vol. 64, pp. 183-190, 2016.

[51] S. Stieglitz, D. Bunker, M. Mirbabaie, and C. Ehnis, "Sensemaking in social media during extreme events," J. Contingencies and Crisis Manage., 2017.

[52] S. Stieglitz and L. Dang-Xuan, "Emotions and Information Diffusion in Social Media-Sentiment of Microblogs and Sharing Behavior,' J. Manage. Inform. Syst., vol. 29, no. 4, pp. 217-248, 2013.

[53] S. Stieglitz, L. Dang-Xuan, A. Bruns, and C. Neuberger, "Social Media Analytics - An Interdisciplinary Approach and Its Implications for Information Systems," Bus. \& Inform. Syst. Eng., vol. 6, no. 2, pp. 89-96, apr 2014.

[54] R. Subba and T. Bui, "Online Convergence Behavior, Social Media Communications and Crisis Response: An Empirical Study of the 2015 Nepal Earthquake Police Twitter Project," in Proc. 50th HICSS, 2017, pp. 284-293.

[55] J. N. Sutton, L. Palen, and I. Shklovski, Backchannels on the front lines: Emergency uses of social media in the 2007 Southern California Wildfires. University of Colorado, 2008.

[56] W. N. Venables and B. D. Ripley, Modern Applied Statistics with $S$, 4th ed. New York: Springer, 2002.

[57] C. Wendling, J. Radisch, and S. Jacobzone, "The Use of Social Media in Risk and Crisis Communication," OECD Working Papers on Public Governance, no. 24, 2013.

[58] J. Yin, A. Lampert, M. Cameron, B. Robinson, and R. Power, "Using Social Media to Enhance Emergency Situation Awareness," IEEE Intelligent Sys., vol. 27, no. 6, pp. 52-59, nov 2012. 